\title{
STRUCTURE OF CERTAIN INDUCED REPRESENTATIONS OF COMPLEX SEMISIMPLE LIE ALGEBRAS ${ }^{1}$
}

\author{
BY DAYA-NAND VERMA ${ }^{2}$
}

Communicated by C. W. Curtis, June 14, 1967

Let $\&$ be a split semisimple Lie algebra over a field $\Phi$ of characteristic zero and $\mathfrak{L}=\mathfrak{H}+\sum_{\alpha \in \Delta} \mathfrak{L}_{\alpha}$ be the rootspace decomposition of $\mathscr{L}$ relative to a splitting Cartan subalgebra $\mathfrak{F}$, where the subset $\Delta$ of $\mathfrak{F C}^{*}$ is the corresponding root-system. Fix a simple system of roots $\left\{\alpha_{1}, \alpha_{2}, \cdots, \alpha_{l}\right\}$, for which the positive (resp. negative) roots are denoted by $\Delta_{+}$(resp. $\Delta_{-}$). For $\alpha \in \Delta$ let $R_{\alpha}$ be the Weyl reflection sending $\alpha$ into $-\alpha$ and fixing the elements of $\mathfrak{F}^{*}$ orthogonal to $\alpha$ with respect to the inverse Killing form $\langle$,$\rangle . It is given explicitly by$ $\lambda R_{\alpha}=\lambda-\lambda\left(h_{\alpha}\right) \alpha$ where $h_{\alpha} \in \mathfrak{F}$ is defined by requiring $\lambda\left(h_{\alpha}\right)$ $=2\langle\alpha, \alpha\rangle^{-1}\langle\lambda, \alpha\rangle$ for all $\lambda \in \mathcal{F C}^{*}$. Denote the group generated by $\left\{R_{\alpha} \mid \alpha \in \Delta\right\}$ by $W$. We abbreviate $R_{\alpha_{i}}$ and $h_{\alpha_{i}}$ by $R_{i}$ and $h_{i}$ respectively. The "simple" reflections $R_{1}, R_{2}, \ldots, R_{l}$ are Coxeter generators of the Weyl group $W$. Let $\mathcal{u}$ be the universal enveloping algebra of $\mathfrak{L}$, and $\mathfrak{U}_{+}$(resp. $\mathcal{U}_{-}$) the subalgebra with identity 1 generated by $\mathfrak{L}_{+}=\sum_{\alpha \in \Delta_{+}} \mathfrak{L}_{\alpha}$ (resp. $\mathfrak{L}_{-}=\sum_{\alpha \in \Delta_{-}} \mathfrak{L}_{\alpha}$ ).

It is an established fact that the notions of $\&$-module and $\mathcal{U}$-module are interchangeable. Here, and throughout, the word "module" is an abbreviation for the word "right-module." Our object in this paper is to study the structure of the $\mathcal{L}$-module $\mathfrak{B}_{\Lambda}=\mathfrak{U} / \mathfrak{d}_{\Lambda}$ for arbitrary $\Lambda \in \mathfrak{H}^{*}$, where $\mathcal{U}$ is regarded as a module under right-multiplication and $\mathfrak{d}_{\Lambda}$ is the right-ideal of $\mathfrak{u}$ (i.e., submodule of $\mathfrak{u}$ ) generated by

$$
\mathscr{L}_{+} \cup\{h-\Lambda(h) \cdot 1 \mid h \in \mathcal{H}\} \text {. }
$$

It is known (cf. Cartier $\left[4\right.$, p. 17-04]) that $\mathfrak{B}_{\Lambda}$ has a unique maximal proper submodule and hence a unique irreducible quotient-module which we denote by $\mathfrak{M}_{\Lambda}$. $\mathfrak{B}_{\Lambda}$ "admits a complete weightspace decomposition" in the sense that it is the direct sum of its weightspaces $\mathfrak{B}_{\Lambda(\lambda)}$, where for any $\mathcal{L}$-module $\mathfrak{M}$ and any $\lambda \in \mathfrak{F}^{*}$ the weightspace $\mathfrak{M}_{(\lambda)}$ is defined by

$$
\mathfrak{M}_{(\lambda)}=\{x \in \mathfrak{M} \mid x h=\lambda(h) x \text { for all } h \in \mathfrak{H C}\} ;
$$

\footnotetext{
${ }^{1}$ Announcement of the results in the author's Ph.D. dissertation, Yale University, 1966: supported in part by the NFS grant no. GP-4017.

2 The author is presently at the Institute for Advanced Study, Princeton, N. J.
} 
and also, $\mathfrak{B}_{\Lambda}$ "has $\Lambda$ as the highest weight" in the sense that $\mathfrak{B}_{\Lambda(\lambda)} \neq 0$ implies $\lambda \leqq \Lambda$. (Here $\lambda \leqq \mu$ means that $\mu-\lambda$ is a nonnegative integral linear combination of the simple roots $\alpha_{1}, \alpha_{2}, \cdots, \alpha_{l .}$ ) These two properties hold for every quotient-module on $\mathfrak{B}_{\Lambda}$, and in particular for $\mathfrak{M}_{\Lambda}$. It is easy to see that $u_{-}$is a direct summand (as a vectorspace) of $g_{\Lambda}$ in $น$. From this, one concludes that $\operatorname{dim} \mathfrak{B}_{\Lambda(\lambda)}=P(\Lambda-\lambda)$ where $P$ is Kostant's partition function. An element of $\mathfrak{B}_{\Lambda}$ is called $\mathfrak{L}_{+}$-extreme if it is annihilated by $\mathfrak{L}_{+}$.

TheOREM 1. Any submodule $\mathfrak{M}$ of $\mathfrak{B}_{\Lambda}$ is generated by the $\mathfrak{L}_{+}$-extreme weight-vectors in $\mathfrak{M}$.

(An element of a weightspace $\mathfrak{M}_{(\lambda)}$ is called a weight-vector of $\mathfrak{M}_{\text {.) }}$

The submodule generated by a nonzero $\mathfrak{L}_{+}$-extreme element of the M-weightspace $\mathfrak{B}_{\Lambda(M)}$ is isomorphic to $\mathfrak{B}_{\mathbf{M}}$. Thus in view of Theorem 1 if one knew all the module-injections $\mathfrak{B}_{M} \rightarrow \mathfrak{B}_{\Lambda}$, one would know all the submodules of $\mathfrak{B}_{\Lambda}$. In fact this knowledge would also settle the inclusion structure of the lattice of submodules of $\mathfrak{B}_{\Lambda}$. (Note that every nonzero homomorphism $\mathfrak{B}_{M} \rightarrow \mathfrak{B}_{\Lambda}$ is injective.)

The action of the center $\mathcal{Z}$ of $\mathcal{U}$ on $\mathfrak{B}_{\Lambda}$ is given by an algebra-homomorphism $\chi_{\Lambda}: \mathcal{Z} \rightarrow \Phi$. In fact, $\chi_{\Lambda}$ is the so-called infinitesimal character. Harish-Chandra $[1$, p. 73] has shown that

$$
\chi_{\mathrm{M}}=\chi_{\Delta} \text { if and only if } \mathrm{M}+\delta=(\Lambda+\delta)^{\sigma} \text { for some } \sigma \in W,
$$

where $\delta$ is half the sum of all the positive roots. (Equivalently $\delta=\lambda_{1}+\lambda_{2}+\cdots+\lambda_{l}$ where $\lambda_{1}, \lambda_{2}$, etc. are such that $\lambda_{i}\left(h_{j}\right)$ is 1 if $i=j$ and 0 otherwise. For another proof of (1) see Cartier [4, p. 19-09]. Cf. also our concluding paragraph.) Hence a necessary condition for the existence of a module-injection $\mathfrak{B}_{\mathbf{M}} \rightarrow \mathfrak{B}_{\Lambda}$ is

$$
\mathrm{M} \leqq \Lambda \text { and } \mathrm{M}=(\Lambda+\delta)^{\sigma}-\delta \text { for some } \sigma \in W .
$$

$\left(\mathrm{M} \leqq \Lambda\right.$ is necessary because otherwise $\mathfrak{B}_{\Lambda(M)}=0$.) Examples show that (2) is not sufficient.

The simplest situation when $\mathfrak{B}_{M} \rightarrow \mathfrak{B}_{\Lambda}$ exists arises when for some $i$

$$
\mathrm{M}=(\Lambda+\delta)^{R_{i}}-\delta \supsetneqq \Lambda .
$$

Then $\Lambda-\mathrm{M}=\left(\Lambda\left(h_{i}\right)+1\right) \alpha_{i}$, and $\mathrm{M} \ \Lambda$ amounts to $\Lambda\left(h_{i}\right)+1$ being a positive integer. Let $f_{i}$ be a fixed nonzero element of $\mathfrak{L}_{-\alpha_{i}}$. Under (3) it is easily seen that

(4) the module-homomorphism of $\mathcal{u}$ into $\mathfrak{u}$ given by the left-multiplication by $f_{i}^{\Lambda\left(h_{i}\right)+1}$ induces an injection of $\mathfrak{B}_{\mathrm{M}}$ into $\mathfrak{B}_{\Lambda}$ (i.e. maps $\mathcal{g}_{\mathrm{M}}$ into $\left.g_{A}\right)$. 
Since $\operatorname{dim} \mathfrak{B}_{\Lambda(M)}=P(\Lambda-M)=1$ (assuming (3)) it is clear that $\mathfrak{B}_{\Lambda}$ contains a unique copy of $\mathfrak{B}_{M}$, i.e., the homomorphism $\mathfrak{B}_{M} \rightarrow \mathfrak{B}_{\Lambda}$ described in (4) is the only one apart from scalar multiples. Even without the hypothesis (3) one has (for arbitrary $\Lambda$ and $M$ ) the following

THEOREM 2. $\mathfrak{B}_{\Lambda}$ never contains more than one copy of $\mathfrak{B} \mathrm{M}$.

The proof is again by counting dimensions, though much more delicate. As a first step it is possible to show that it suffices to prove Theorem 2 in case $\mathfrak{B} \mathrm{M}$ is irreducible. Then one notices that if in that case this theorem were to be false one would have

$$
P(\Lambda-\lambda)=\operatorname{dim} \mathfrak{B}_{\Lambda(\lambda)} \geqq 2 \operatorname{dim} \mathfrak{B}_{M(\lambda)}=2 P(M-\lambda) \quad \text { for all } \lambda \in \mathcal{F C}^{*} \text {. }
$$

But this is seen to be impossible on putting $\lambda_{0}=\Lambda-M$ and $\mu=M-\lambda$ in the following.

Lemma 3. Given $\lambda_{0} \in \mathfrak{F}^{*}$, one can find $\mu$ such that

$$
P\left(\lambda_{0}+\mu\right)<2 P(\mu) \text {. }
$$

The proof of this is based on the fact that $P(\lambda)$ is bounded by a polynomial function of $\lambda$.

Since for any given $\Lambda$ there are only a finite number of $M$ satisfying (2), it is clear from Theorems 1 and 2 that $\mathfrak{B}_{\Lambda}$ has only a finite number of submodules. In fact for a "general" $\Lambda, \mathfrak{B}_{\Lambda}$ is itself irreducible. Specifically, this is so in case $\Lambda\left(h_{\alpha}\right)$ is a noninteger for each $\alpha \in \Delta$.

Let $\mathfrak{D}\left(\mathcal{U}_{-}\right)$denote the division ring of quotients of $\mathcal{u}_{-}[2$, p. 166].

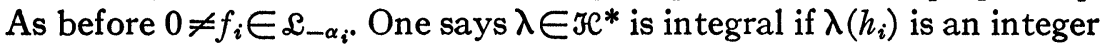
for $1 \leqq i \leqq l$; then $\lambda^{\sigma}$ is also integral for $\sigma \in W$. The following is a key result.

TheOREM 4. Let $\lambda$ be integral, and $\left(i_{1}, i_{2}, \cdots, i_{k}\right)$ be a sequence drawn from the set $\{1,2, \cdots, l\}$. The element $f_{i_{1}}^{n_{1}} f_{i_{2}}^{n_{2}} \cdots f_{i_{k}}^{n_{k}}$ of $\mathfrak{D}\left(\mathcal{U}_{-}\right)$where the integers $n_{1}, n_{2}, \cdots, n_{k}$ are defined by $n_{q}=\left(\lambda R_{i_{1}}, R_{i_{2}} \cdots R_{i_{q-1}}\right)$ $\left(h_{i_{q}}\right)$, depends only on $\lambda$ and the product $\sigma=R_{i_{1}} R_{i_{2}} \cdots R_{i_{k}}$ and not on the choice of the sequence. Denote this element by $u(\lambda, \sigma)$.

(For typographical convenience $\lambda^{\tau}$ is also written as $\lambda \tau$.) It suffices to prove the theorem for dominant integral $\lambda$ (i.e., when $\lambda\left(h_{i}\right)$ is a nonnegative integer for each $i$ ) and those sequences that are reduced (i.e., for which $\sigma$ can not be written as a product of less than $k$ simple reflections). It is easily seen that then $n_{1}, n_{2}, \cdots, n_{k}$ are all nonnegative integers. Applying (4) $k$ times for $\lambda-\delta, \lambda R_{1}-\delta, \lambda R_{1} R_{2}-\delta, \cdots$ etc. in place of $\Lambda$, it is easily seen that the left-multiplication $L_{u}$ by 
$u=f_{t_{1}}^{n_{1}} f_{t_{2}}^{n_{2}} \cdots f_{t_{k}}^{n_{k}}$ induces an injection of $\mathfrak{B}_{\mathrm{M}}$ into $\mathfrak{B}_{\Lambda}$ where $\Lambda=\lambda-\delta$ and $M=\lambda^{\sigma}-\delta$. Thus in view of Theorem 2 if another sequence $\left(j_{1}, j_{2}, \cdots, j_{k}\right)$ satisfying $\sigma=R_{j_{1}} R_{j_{2}} \cdots R_{j_{k}}$ yields $u^{\prime}$ in place of $u$, then $L_{u}=c L_{u^{\prime}}$ for some scalar $c$. That gives $u=c u^{\prime}$. To show that in all cases $c=1$, it suffices to do so when $\mathscr{L}$ is simple of rank 2 , and $\sigma$ is that unique element of $W$ which sends $\Delta_{+}$into $\Delta_{\text {- }}$. This is because $W$ is a Coxeter group. For each of the three possibilities for 2 , viz., $A_{2}, B_{2}$ or $G_{2}, \sigma$ can be written in two different ways with $k$ equal to 3,4 or 6 respectively. Thus

$$
\text { for } A_{2}: f_{1}^{m} f_{2}^{m+n} f_{1}^{n}=f_{2}^{n} f_{1}^{m+n} f_{2}^{m},
$$

where $m=\lambda\left(h_{1}\right)$ and $n=\lambda\left(h_{2}\right)$. In the same way

$$
\begin{aligned}
& \text { for } B_{2}: f_{1}^{m} f_{2}^{m+n} f_{1}^{m+2 n} f_{2}^{n}=f_{2}^{n} f_{1}^{m+2 n} f_{2}^{m+n} f_{1}^{m} ; \text { and } \\
& \text { for } G_{2}: f_{1}^{m} f_{2}^{m+n} f_{1}^{2 m+8 n} f_{2}^{m+2 n} f_{1}^{m+8 n} f_{2}^{n}=f_{2}^{n} f_{1}^{m+3 n} f_{2}^{m+2 n} f_{1}^{2 m+8 n} f_{2}^{m+n} f_{1}^{m} .
\end{aligned}
$$

Here for $B_{2}$ and $G_{2}$ we have taken $\alpha_{1}$ to be the shorter simple root; since the two simple roots have unequal length, the statements (6) and (7) are not symmetrical in the indices 1 and 2.

(5) is easy to prove $a b$ initio. In (6) and (7) let $u$ and $u^{\prime}$ be respectively the left and right sides. We saw that $u=c u^{\prime}$. But it can be shown that the action of $u$ and $u^{\prime}$ is the same, and is not zero, on a highest weight-vector of the finite-dimensional \&-module with highest weight $m \lambda_{1}+n \lambda_{2}$. Hence $c=1$. It may be remarked that (6) can also be proved directly (i.e., without using $u=c u^{\prime}$ ) though clumsily. But no such direct proof of (7) is known. Thus if no simple component of $\&$ is of type $G_{2}$, Theorem 4 can be proved without invoking a difficult result, Theorem 2, on infinite-dimensional \&-modules.

THEOREM 5. For integral $\Lambda, \mathfrak{B}_{\Lambda}$ contains a copy of $\mathfrak{B}_{\mathrm{M}}$ for $\mathrm{M}$ $=(\Lambda+\delta)^{\sigma}-\delta$ if and only if $u(\Lambda+\delta, \sigma)$ (defined in Theorem 4$)$ is in $u_{-}$.

Note that this criterion does not make sense for $\Lambda$ nonintegral. On the positive side we have the following result which is hard to prove, and should be regarded our deepest finding. This generalizes (4).

TheOREM 6. Let $\alpha \in \Delta$ and suppose $(\Lambda+\delta)\left(h_{\alpha}\right)$ is a nonnegative integer. If $\Lambda$ is integral, $u\left(\Lambda+\delta, R_{\alpha}\right)$ is in $u_{-}$, so that $\mathfrak{B}_{\Lambda}$ contains a copy of $\mathfrak{B}_{\mathrm{M}}$ for $\mathbf{M}=(\Lambda+\delta) R_{\alpha}-\delta=\Lambda-(\Lambda+\delta)\left(h_{\alpha}\right)$; the latter conclusion is valid, however, even if $\Lambda$ is not integral.

It follows that the following condition on $\Lambda$ and $M$ ensures the existence of a copy of $\mathfrak{B}_{\mathrm{M}}$ in $\mathfrak{B}_{\Lambda}$ : 
there exist positive roots $\gamma_{1}, \gamma_{2}, \cdots, \gamma_{m}$ such that

$\Lambda=\Lambda_{0} \geqq \Lambda_{1} \geqq \Lambda_{2} \geqq \cdots \geqq \Lambda_{m}=M$

holds where $\Lambda_{q}$ is recursively defined to be

$\left(\Lambda_{q-1}+\delta\right) R_{\gamma_{q}}-\delta$ for $1 \leqq q \leqq m$.

This sufficient condition should be contrasted with the necessary condition (2). If for a given $\Lambda$ the two conditions on $\mathbf{M}$ coincide one knows all submodules of $\mathfrak{B}_{\Lambda}$. This is precisely what happens when $\mathfrak{L}$ is of rank 2 and $\Lambda$ is integral. Examples show that in general (8) does not coincide with (2) even for integral $\Lambda$.

CONJECTURE 1. The condition (8) is also necessary for $\mathfrak{B}_{\Lambda}$ to contain a copy of $\mathfrak{B}_{\mathrm{M}}$.

An affirmative answer to this has interesting consequences which we proceed to discuss. The following is an easy initial step.

THEOREM 7. The composition factors of $\mathfrak{B}_{\Lambda}$ are all nonisomorphic and consist of $\mathfrak{M}_{\mathbf{M}}$ for all those $\mathrm{M}$ for which $\mathfrak{B}_{\mathbf{\Lambda}}$ contains a copy of $\mathfrak{B}_{\mathbf{M}}$.

Now we introduce the notation $\Lambda_{\sigma}$ to stand for $(\Lambda+\delta)^{\sigma}-\delta$. Clearly $\left(\Lambda_{\sigma}\right)_{\tau}=\Lambda_{\sigma \tau}$. For sake of simplicity from now on we restrict our attention to the situation where $\Lambda$ is integral and $\Lambda_{\sigma} \neq \Lambda$ whenever $\sigma \neq \mathrm{id}$. To suit this situation it is more convenient, however, to replace $\Lambda$ and $M$ in the preceding by $\Lambda_{\sigma}$ and $\Lambda_{\tau}$ where now $\Lambda$ is dominant integral; this can be done for unique $\Lambda, \sigma$ and $\tau$ under the assumption of the last sentence and (2). The condition (8) then becomes a condition on $\sigma$ and $\tau$ independent of (our new) $\Lambda$ :

$$
\begin{aligned}
& \text { there exist positive roots } \gamma_{1}, \gamma_{2}, \cdots, \gamma_{m} \\
& \text { such that } \tau=\sigma R_{\gamma_{1}} R_{\gamma_{2}} \cdots R_{\gamma_{m}} \text { and } \\
& \gamma_{Q} \in \Delta_{+} \sigma R_{\gamma_{1}} R_{\gamma_{2}} \cdots R_{\gamma_{-1}} \text { for } 1 \leqq q \leqq m .
\end{aligned}
$$

We use this condition to define a partial-ordering on $W$, and write $\sigma \prec \tau$ (equivalently $\tau \succ \sigma$ ) in place of (9). The identity is the lowest element in this ordering on $W$, and the element $\sigma_{0}$ which carries $\Delta_{+}$ into $\Delta_{-}$is the highest. It can be seen that if $\tau=R_{i_{1}} R_{i_{2}} \cdots R_{i_{k}}$ is a reduced expression for $\tau$, then $\sigma \prec \tau$ if and only if $\sigma=R_{j_{1}} R_{j_{2}} \cdots R_{j_{p}}$ where $\left(j_{1}, j_{2}, \cdots, j_{p}\right)$ is a subsequence of $\left(i_{1}, i_{2}, \cdots, i_{k}\right)$. [This ordering also arises from the Bruhat decomposition as follows: Let $B$ denote a Borel subgroup of the complex adjoint group of $\mathcal{L}$, and the manifold $G / B$ be given the cell-decomposition $\bigcup_{\sigma \in W} C_{\sigma}$ where $C_{\sigma}$ is the $B$-orbit on $G / B$ of the coset of an element of $G$ whose restriction to $\mathcal{H C}$ coincides with the action of $\sigma$ on $\Re$. Then $\sigma \prec \tau$ if and only if $C_{\sigma}$ is contained in the closure of $\boldsymbol{C}_{\boldsymbol{\tau}}$.] 
Thus, Conjecture 1 says that $\mathfrak{B}_{\Lambda_{\sigma}}$ contains a copy of $\mathfrak{B}_{\Lambda_{\tau}}$ if and only if $\sigma \prec \tau$. Remember that now $\Lambda$ is dominant integral. Let us denote the dimension of the $\lambda$-weightspace of the irreducible module $\mathfrak{M}_{\Lambda_{\text {, }}}$ by $m_{\tau}(\lambda)$. It follows from Theorem 7 , modulo the truth of the conjecture, that

$$
\sum_{\tau>\sigma} m_{\tau}(\lambda)=\operatorname{dim} \mathfrak{B}_{\Lambda_{\sigma}(\lambda)}=P\left(\Lambda_{\sigma}-\lambda\right) .
$$

Thus for each fixed $\lambda,(10)$ gives $|W: 1|$ linear relations among the same number of unknowns $m_{\tau}(\lambda), \tau \in W$. We ought to consider this set of unknowns as a function $\phi$ on $W$ (with value $m_{\tau}(\lambda)$ at $\tau$ ); then (10) gives the partial-sum function $\psi(\sigma)=\sum_{\tau \geqslant \sigma} \phi(\tau)$ on $W$. Thus solving the linear equation (10) (for each fixed $\lambda$ ) amounts to inverting the partial-sum operator that sends $\phi$ into $\psi$. It is known [3 p. 344] that this operator is indeed invertible: $\phi(\kappa)=\sum_{\sigma>\kappa} \mu(\kappa, \sigma) \psi(\sigma)$ where $\mu(\kappa, \tau)$ is the so-called Möbius function of the partial-ordering on $W$ defined by the requirement that $\sum_{\tau>0>x} \mu(\kappa, \sigma)$ be 1 or 0 according as $\tau=\kappa$ or $\tau \neq \kappa$. We now conjecture that $\mu(\kappa, \sigma)=\operatorname{det} \kappa \sigma$ which is clearly equivalent to

Conjecture 2. $\tau \neq \kappa$ implies $\sum_{\tau>\sigma}{ }_{\kappa} \operatorname{det} \sigma=0$.

Assuming the truth of this conjecture also, one has, on solving (10), the following formula for the weight-multiplicity of $\mathfrak{M}_{\Lambda_{\boldsymbol{k}}}$ :

$$
m_{\kappa}(\lambda)=\sum_{\sigma>x} \operatorname{det}(\kappa \sigma) \cdot P\left((\Lambda+\delta)^{\sigma}-\lambda-\delta\right) .
$$

When $\kappa$ is the identity element of $W$ this becomes the multiplicity formula of Kostant for the finite-dimensional representation $\mathfrak{M}_{\Delta}$. It is well known that the celebrated character formula of $\mathrm{H}$. Weyl is a trivial consequence of Kostant's formula.

It may appear that Weyl's character formula has been used in our development right at the first step, i.e., through the known proofs of (1) - the theorem of Harish-Chandra. Fortunately, we have been able to supply a new and self-contained proof of (1). For this first observe that the "if" part of (1) is an easy consequence of (4) at least for integral $\Lambda$; transition from integral to arbitrary $\Lambda$ is easy on the basis of an (algebraic) continuity argument. The burden of the proof of the difficult "only if" part is transferred to the somewhat easier theorem of Chevalley which says that the restriction of the G-invariant polynomials on $\mathscr{E}$ to $\mathcal{H}$ is onto the space of $W$-invariant polynomials on $\mathcal{H}$. An easy and direct proof of Chevalley's theorem is due to Kostant and Steinberg. This line of proof of (1) was independently 
arrived at by K. R. Parthasarathy, R. Ranga Rao and V. S. Varadarajan (unpublished).

\section{REFERENCES}

1. Harish-Chandra, On some applications of the universal enveloping algebra of a semisimple Lie algebra, Trans. Amer. Math. Soc. 70 (1951), 28-96.

2. N. Jacobson, Lie algebras, Interscience, New York, 1962.

3. G.-C. Rota, On the foundations of combinatorial theory. I. Theory of Möbius functions, Zeits. für Wahrscheinlichkeitstheorie 2 (1964), 340-368.

4. Seminaire "Sophus Lie" de l'Ecole Norm. Sup., 1954/1955, Vol. 1, Secretariat Mathematique, Paris.

YALE UNIVERSITY 